\title{
Increased Synchrony and Bursting of Dorsal Cochlear Nucleus Fusiform Cells Correlate with Tinnitus
}

\author{
Calvin Wu, ${ }^{1}$ David T. Martel, ${ }^{1,2}$ and ${ }^{-S}$ Susan E. Shore ${ }^{1,2,3}$ \\ ${ }^{1}$ Kresge Hearing Research Institute, Department of Otolaryngology, and Departments of ${ }^{2}$ Biomedical Engineering and ${ }^{3}$ Department of Molecular and \\ Integrative Physiology, University of Michigan, Ann Arbor, Michigan 48109
}

Tinnitus, the perception of phantom sounds, is thought to arise from increased neural synchrony, which facilitates perceptual binding and creates salient sensory features in the absence of physical stimuli. In the auditory cortex, increased spontaneous cross-unit synchrony and single-unit bursting are de facto physiological correlates of tinnitus. However, it is unknown whether neurons in the dorsal cochlear nucleus (DCN), the putative tinnitus-induction site, exhibit increased synchrony. Using a temporary-threshold shift model and gap-prepulse inhibition of the acoustic startle to assess tinnitus, we recorded spontaneous activity from fusiform cells, the principle neurons of the DCN, in normal hearing, tinnitus, and non-tinnitus guinea pigs. Synchrony and bursting, as well as spontaneous firing rate (SFR), correlated with behavioral evidence of tinnitus, and increased synchrony and bursting were associated with SFR elevation. The presence of increased synchrony and bursting in DCN fusiform cells suggests that a neural code for phantom sounds emerges in this brainstem location and likely contributes to the formation of the tinnitus percept.

Key words: bursting; dorsal cochlear nucleus; fusiform cells; neural synchrony; spontaneous activity; tinnitus

\section{Significance Statement}

Tinnitus, a phantom auditory percept, is encoded by pathological changes in the neural synchrony code of perceptual processing. Increased cross-unit synchrony and bursting have been linked to tinnitus in several higher auditory stations but not in fusiform cells of the dorsal cochlear nucleus (DCN), key brainstem neurons in tinnitus generation. Here, we demonstrate increased synchrony and bursting of fusiform cell spontaneous firing, which correlate with frequency-specific behavioral measures of tinnitus. Thus, the neural representation of tinnitus emerges early in auditory processing and likely drives its pathophysiology in higher structures.

\section{Introduction}

Tinnitus, or auditory phantom perception, arises from alterations in sound encoding in the central auditory pathway (Eggermont, 2015). This pathological state is often, but not always, triggered by auditory insult (König et al., 2006) and manifests as increased resting-state neural activity and synchronization in the human primary auditory (A1) and associated cortices (Elgoyhen et al., 2015). Thus, hyperactivity and synchrony may encode sound representations in tinnitus (Weisz et al., 2007). Because features of an auditory stimulus are extracted by assemblies of

Received Nov. 2, 2015; revised Jan. 6, 2016; accepted Jan. 10, 2016.

Author contributions: C.W., D.T.M., and S.E.S. designed research; C.W. and D.T.M. performed research; C.W., D.T.M., and S.E.S. analyzed data; C.W., D.T.M., and S.E.S. wrote the paper.

This study was supported by National Institutes of Health Grants R01-DC004825 (S.E.S.) and T32-DC00011 (C.W.) and the Wallace H. Coulter Translational Research Partnership. We thank James Wiler and Chris Ellinger for technical assistance and Michael Roberts, Sandy Bledsoe, and Amarins Heeringa for helpful comments.

The authors declare no competing financial interests.

Correspondence should be addressed to Dr. Susan E. Shore, Department of Otolaryngology, University of Michigan, 1150 West Medical Center Drive, Ann Arbor, MI 48109. E-mail: sushore@umich.edu.

DOI:10.1523/JNEUROSCI.3960-15.2016

Copyright $\odot 2016$ the authors $\quad 0270-6474 / 16 / 362068-06 \$ 15.00 / 0$ neurons firing synchronously, a hypersynchronous neural ensemble could signal the presence of "auditory objects" even in the absence of external stimuli, giving rise to the tinnitus percept.

The substructure of neural synchronization is spontaneous cross-unit synchrony and single-unit bursting (Singer, 1993). In animal models of noise trauma (Noreña and Eggermont, 2003) and tinnitus (Engineer et al., 2011), correlates of increased synchrony and bursting have been confirmed in A1. However, lower stations also exhibit increased synchrony and bursting as correlates of tinnitus: bursting in the medial geniculate body (Kalappa et al., 2014) and synchrony and bursting in the inferior colliculus (IC; Bauer et al., 2008). The presence of increased synchrony and bursting in subcortical nuclei suggests that perceptual binding by means of synchronous neural activity is processed early and diffusely in the auditory pathway.

The dorsal cochlear nucleus (DCN), a brainstem nucleus that receives direct input from the auditory nerve, is essential for tinnitus induction (Kaltenbach, 2011). Fusiform cells, the principal output neurons of the DCN, exhibit increased spontaneous firing rates (SFRs) after noise trauma and tinnitus (Brozoski et al., 2002; Kaltenbach et al., 2004; Dehmel et al., 2012), which are driven by 
homeostatic and timing-dependent plasticity (Koehler and Shore, 2013; Li et al., 2015). Increased SFR, an established tinnitus correlate, is unperturbed by transection of afferent or efferent input to the DCN (Zacharek et al., 2002; Zhang et al., 2006), whereas bilateral DCN ablation prevents the development of tinnitus (Brozoski et al., 2002). However, despite evidence for their essential role in tinnitus induction, the presence of increased synchrony in fusiform cells remains unexplored. As increased synchrony encodes for tinnitus, it is plausible that the neural representation of tinnitus originates in fusiform cells.

Consistent with this hypothesis, we showed that synchrony, bursting, and SFRs of fusiform cells correlate with behavioral evidence of tinnitus in guinea pigs. In tinnitus animals, physically and tonotopically adjacent unit pairs showed increased cross-correlations associated with SFR, whereas synchrony first appeared in a group of unit pairs separated by larger distances. In addition, increased bursting was driven by the increased SFR and accompanied by increases in nonbursting spikes. These findings reveal that degraded perceptual processing occurs in DCN fusiform cells and likely influences tinnitus pathophysiology in higher-order structures.

\section{Materials and Methods}

Animals. All animal procedures were performed in accordance with protocols established by the National Institutes of Health (Publication 8023) and approved by the University Committee on Use and Care of Animals at the University of Michigan. Female, pigmented guinea pigs $(n=14)$ were obtained from Elm Hill Labs at 2-3 weeks of age.

Noise exposure and auditory brainstem responses. Ten guinea pigs were exposed to narrow-band noise to produce a temporary threshold shift (TTS) for tinnitus induction (Dehmel et al., 2012). Four guinea pigs served as sham-exposed (anesthesia-only) controls. Noise exposures and auditory brainstem response (ABR) recordings to assess hearing thresholds were performed in a double-walled soundproof booth. Guinea pigs were anesthetized with ketamine/xylazine $[40 \mathrm{mg} / \mathrm{kg}$ ketamine (Putney); $10 \mathrm{mg} / \mathrm{kg}$ xylazine (Lloyd)]. Sound exposures were unilateral via microphone inserts (Brüel and Kjær) with a $7 \mathrm{kHz}$ centered, half-octave noise at $97 \mathrm{~dB}$ SPL for $2 \mathrm{~h}$. A second noise/sham exposure was performed after 4 weeks. ABRs ( $0-90 \mathrm{~dB}$ SPL tone bursts; $2 \mathrm{~ms} \cos ^{2}$ rise/fall times, $10 \mathrm{~ms}$ duration, $30 \mathrm{~Hz}$ presentation rate, 1024 repetitions in $10 \mathrm{~dB}$ steps; Tucker-Davis Technologies) were recorded at 8, 12, and $16 \mathrm{kHz}$, before, immediately after, and 1 week after each noise exposure.

Behavioral tinnitus assessment. Gap-prepulse inhibition of the acoustic startle (GPIAS) was used to assess tinnitus (Turner et al., 2006). A constant background carrier (band limited at $8-10,12-14$, and $16-18 \mathrm{kHz}$ ) was presented at $65 \mathrm{~dB}$ SPL. Startle responses elicited by broadband noise pulses ( 2 $\mathrm{ms}$ rise/fall time, $20 \mathrm{~ms}$ ) at $95 \mathrm{~dB}$ SPL were quantified by video tracking (Point Gray Research) of the Preyer reflex (modified from Berger et al., 2013). All animals showed stable startle reflex amplitudes over the duration of the study. Startle reflexes were inhibited by a $50 \mathrm{~ms}$ silent gap or $75 \mathrm{~dB}$ SPL prepulse (with equivalent $5 \mathrm{~ms}$ onset/offset time) $100 \mathrm{~ms}$ before the startle pulse. A normalized inhibition ratio $(R)$ was computed by dividing the mean startle amplitude for the gap (or prepulse) trials by those for the startle-only, or no-gap, trials. $R$ values were pooled over the 4 week, twice weekly sessions to establish a behavioral baseline. Four weeks after the second noise exposure, postexposure $R$ values for each animal were calculated. In this study, $R$ values for prepulse inhibition were unchanged before and after noise exposure. An animal was presumed to have tinnitus if the postexposure mean $R$ value for gap inhibition was significantly greater than the baseline value ( $\alpha=$ 0.05 ). The changes in gap $R$ values from preexposure to postexposure were quantified by the standardized tinnitus index $[(x-\mu) / \sigma]$ (Kalappa et al., 2014 ), where $x$ is the postexposure gap $R$ value, and $\mu$ and $\sigma$ are the mean and $\mathrm{SD}$ of the preexposure gap $R$ value. A larger positive index indicates a higher degree of impaired gap detection ("more tinnitus") after noise exposure.

Single-unit recordings. Neurophysiological recordings were performed 8 weeks after the first noise exposure. Animals were anesthetized and secured in a stereotaxic frame with hollow ear bars (David Kopf Instru- ments). The surface of the cerebellum was exposed after a small craniotomy. A two-shank, 16-channel probe (NeuroNexus) was inserted stereotaxically through an intact cerebellum. Recorded voltages were digitized by a PZ2 preamplifier (Tucker-Davis Technologies) and bandpass filtered $(0.3-3 \mathrm{kHz})$ for spike detection, with threshold set at $2.5 \mathrm{SDs}$ above background noise. Broadband noise bursts were used to locate units in the DCN. Receptive fields $(0.1-24 \mathrm{kHz} ; 0-90 \mathrm{~dB})$ were recorded to determine thresholds and best frequencies (BFs). A suitable electrode location in the fusiform cell layer was confirmed by robust responses to BF tones with buildup or pauser-buildup temporal patterns typical of fusiform cells. Spontaneous activity was recorded for $153 \mathrm{~s}$.

Neural synchrony analyses. Recorded spikes were sorted manually into single units using the Plexon Offline Sorter and imported into MATLAB as timestamps. Spatial synchrony was calculated using paired crosscorrelation (Voigt and Young, 1990; Noreña and Eggermont, 2003). Common spikes occurring within $\pm 150 \mu$ s across different units were removed. Cross-correlation coefficients $[p(\tau)]$ were computed as a function of time lag for each pairwise combination of spike trains (Eq. 1):

$$
p(\tau)=\frac{R_{A B}(\tau)-E}{\sqrt{N_{A} N_{B}}}, \quad E=\frac{N_{A} N_{B}}{n},
$$

where $R_{A B}(\tau)$ is the unbiased cross-correlation of spike trains $A$ and $B$, and $N_{A}$ and $N_{B}$ indicate spike counts in the respective spike trains. $E$ is the expectancy of coincidence for Poisson-distributed data (Eq. 2), defined by the multiplication of $N_{A}$ and $N_{B}$ over the number of bins $(n)$. Bin size was constant at $0.3 \mathrm{~ms}$ (Voigt and Young, 1990). A unit pair was considered synchronous when the peak $p$ value was greater than \pm 4 SDs from the mean $p(\tau)$. In the present study, both significant positive and negative cross-correlations were observed, but only positive peak $p$ values were considered in additional analyses.

Bursting was characterized using the Poisson surprise method (Noreña and Eggermont, 2003). The negative log-likelihood (S) measures how unlikely, or "surprising," a series of spikes is within a Poissondistributed spike train (Eq. 3):

$$
S=-\log \left(e^{-r T} \sum_{i=\mathrm{n}}^{\infty} \frac{(r T)^{i}}{i !}\right),
$$

where $r$ is the mean SFR of the spike train, and $T$ is a given time interval that contains $n$ spikes. $S$ values were computed for every three spikes occurring in interval $T(n+2)-T(n)$, and the triplet was considered a burst when its $S$ value exceeded 10. When a burst was identified, additional spikes $(T(n+3), T(n+4) \ldots)$ were recruited to increase burst duration until the $S$ value decreased. In addition, the first spike $(n)$ in a burst was removed if the resulting new burst returned a higher $S$ value. Each defined burst contains information regarding its structure: $S$ value, on/off time, and number of spikes incorporated.

Statistics. Statistical tests, including Pearson's linear correlation, analysis of covariance (ANCOVA), and ANOVAs were used to determine statistical differences $(\alpha=0.05)$. Post hoc analyses were performed using the Tukey-Kramer method.

\section{Results}

ABR thresholds (Fig. 1A) of noise-exposed animals exhibited 20-40 dB elevations immediately after exposure (Fig. 1B; twoway repeated-measures ANOVA, $\left.F_{(2,2)}=12.3, p=0.0006\right)$ but recovered to the preexposure baseline within 1 week (post hoc, $p>0.05)$. A second noise exposure produced similar TTSs. Unexposed, control animals did not show ABR threshold shifts (Fig. $1 C$ ), and neither exposed nor control animals showed permanent threshold shifts. Eight weeks after the first exposure, six animals $(60 \%)$ showed behavioral evidence of tinnitus with significantly higher tinnitus indices than unexposed controls and the other four animals (40\%) without behavioral signs of tinnitus (Fig. 1D; two-way ANOVA, $F_{(2,2)}=1.12, p=0.0025$ for tinnitus classification), predominantly at 12 and $16 \mathrm{kHz}$ (post hoc, $p<0.05$ ). There was no correlation between the amount of threshold 
shift and resulting tinnitus (Fig. $1 E: F_{(2,1)}$ $=2.52, p=0.13$; Fig. $1 F: r_{(32)}^{2}=0.29, p=$ $0.10)$. To test for potential suprathreshold hearing impairment as a result of TTSs (Kujawa and Liberman, 2009), we measured ABR wave I amplitudes for suprathreshold intensities (Fig. 1A) in tinnitus and non-tinnitus animals. There was no significant wave I decrement after recovery from TTS in either group (Fig. $1 G$; three-way ANOVA, $F_{(116,1,1)}=0.12, p=$ 0.73 for exposure time point, $F_{(116,1,1)}=$ $2.89, p=0.12$ for tinnitus classification). There was also no correlation between wave I amplitude (at $80 \mathrm{~dB}$ ) and tinnitus index (Fig. 1H: $F_{(2,2)}=0.02, p=0.70$; Fig. 1I: $\left.r_{(25)}^{2}=0.13, p=0.52\right)$.

Physiological differences between tinnitus and non-tinnitus animals were apparent in fusiform cell spontaneous activity. Synchrony, quantified by the peak cross-correlation coefficient between fusiform cell pairs (Fig. 2A, inset), was elevated in tinnitus animals compared with the unexposed controls and non-tinnitus animals (Fig. $2 A$; two-way ANOVA, $F_{(4,2)}=3.5, p=0.03$ for tinnitus classification) and was highest in tonotopic regions $\sim 1$ octave above the frequency of noise-exposure peak intensity (post hoc, $p<0.05$ ). Synchrony correlated with tinnitus behavior at corresponding 8 , 12 , and $16 \mathrm{kHz}$ bins $\left(r_{(374)}^{2}=0.15, p=\right.$ 0.0048; Fig. 2B).

In addition to increased peak crosscorrelation coefficients, both the Euclidean distance (physical distance between recording sites) and the BF difference between units (tonotopic distance) decreased in tinnitus animals (Fig. 2C: $r_{(443)}^{2}=-0.10, p=0.042$; Fig. $2 D: r_{(452)}^{2}=$ $-0.09, p=0.044)$. Plotting distance measurements to peak crosscorrelation coefficients (Fig. 2E) revealed two patterns of increased synchrony in tinnitus animals: unit pairs with short physical and tonotopic distances showed increased cross-correlation strengths [peak cross-correlation coefficient; Fig. $2 E$, (a)], governing the anticorrelation with tinnitus index, whereas a distinct population of unit pairs with high tonotopic and physical distances (unsynchronized in normal and no-tinnitus animals) became synchronized in tinnitus animals [Fig. 2E, (b)].

Spontaneous bursting also increased in tinnitus animals (Fig. $3 A ; F_{(6,2)}=16.9, p=6.2 \times 10^{-8}$ for tinnitus classification; $F_{(6,2)}$ $=3.3, p=9.3 \times 10^{-5}$ for interaction). Significant increases in burst rate were observed in frequency bins $(12-16 \mathrm{kHz})$ with high tinnitus indices (Fig. 1C). Interestingly, non-tinnitus units showed significantly lower burst rates than both the unexposed controls and tinnitus animals in this region (post hoc, $p<0.05$ ). Burst rate and percentage bursting time correlated positively with tinnitus behavior (Fig. $3 B: r_{(710)}^{2}=0.22, p=6.3 \times 10^{-9}$; Fig. $3 C$ : $\left.r_{(797)}^{2}=0.14, p=4.9 \times 10^{-5}\right)$, whereas average burst duration correlated negatively with tinnitus behavior (Fig. $3 D ; r_{(414)}^{2}=-0.25$, $\left.p=2.3 \times 10^{-7}\right)$. Decreased burst duration combined with increased burst rate (Fig. $3 E$ ) suggests that the burst pattern is reorganized in tinnitus.
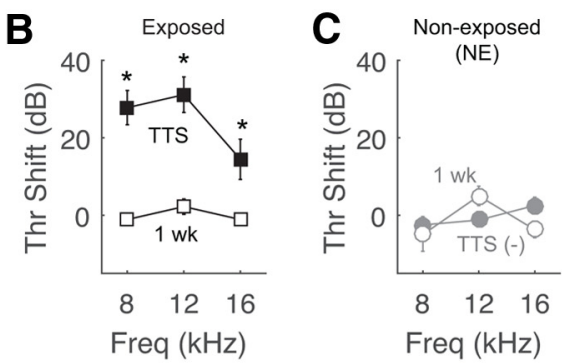

$\mathbf{F}$
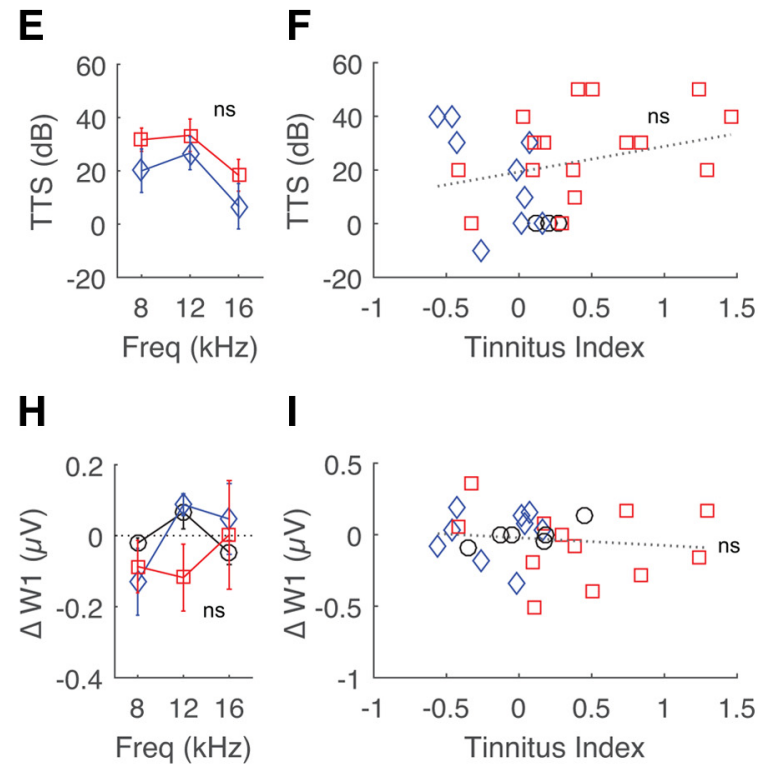

Figure 1. Noise exposure results in distinct populations of animals with and without tinnitus. $\boldsymbol{A}$, Representative $A B R$ waveforms showing wave I amplitude (W1) and threshold determination. $\boldsymbol{B}, \boldsymbol{C}, \mathrm{ABR}$ threshold shifts at 8, 12, and $16 \mathrm{kHz}$ in exposed or ET and ENT animals before and 1 week after exposure at all frequencies. $\boldsymbol{H}$, Changes in W1 at $80 \mathrm{~dB}(\Delta W 1)$ across frequencies. $\boldsymbol{I}$, Correlation of $\Delta \mathrm{W} 1$ and tinnitus index at all frequencies.

Because synchrony and bursting both correlate with tinnitus behavior, they may simply reflect the SFR elevation associated with tinnitus (Brozoski et al., 2002; Kaltenbach et al., 2004; Dehmel et al., 2012). As expected, increased SFR was observed in tinnitus above the peak exposure frequency $\left(F_{(7,2)}=20.0, p=3.2 \times 10^{-9}\right.$ for tinnitus classification; $F_{(7,2)}=2.1, p=0.0088$ for tinnitus class $\times$ frequency interaction; Fig. $4 A$ ) and correlated with tinnitus behavior (Fig. $4 B$; $\left.r_{(736)}^{2}=0.21, p=1.7 \times 10^{-8}\right)$. However, synchrony did not correlate directly with SFR [Fig. 4C, (a); $r_{(444)}^{2}=0.12, p=0.07$ ] because of the influence of unit pairs with increased distances but low crosscorrelation strengths [Figs. $2 E$, (b), $4 C$, filled). When only shortdistance unit pairs $(<200 \mu \mathrm{m})$ were analyzed, a significant correlation with SFR was observed [Fig. $4 C$, (b), open; $r_{(112)}^{2}=0.21$, $p=0.026]$. Similarly, burst rate showed a linear relationship with SFR (Fig. $4 D ; r_{(948)}^{2}=0.87, P \rightarrow 0$ ), although the linear slope for tinnitus units was significantly shallower (ANCOVA, $F_{(2,898)}=$ $\left.119.3, p=1.1 \times 10^{-46}\right)$, reflecting the altered burst pattern (Fig. $3 E$ ). Thus, although synchrony and bursting are linked to SFR, they contribute additional tinnitus phenotypes of lengthened synchrony distance and decreased burst duration.

\section{Discussion}

This study reveals that increased synchrony and bursting in fusiform cells correlate with behavioral evidence of tinnitus. The physiological 

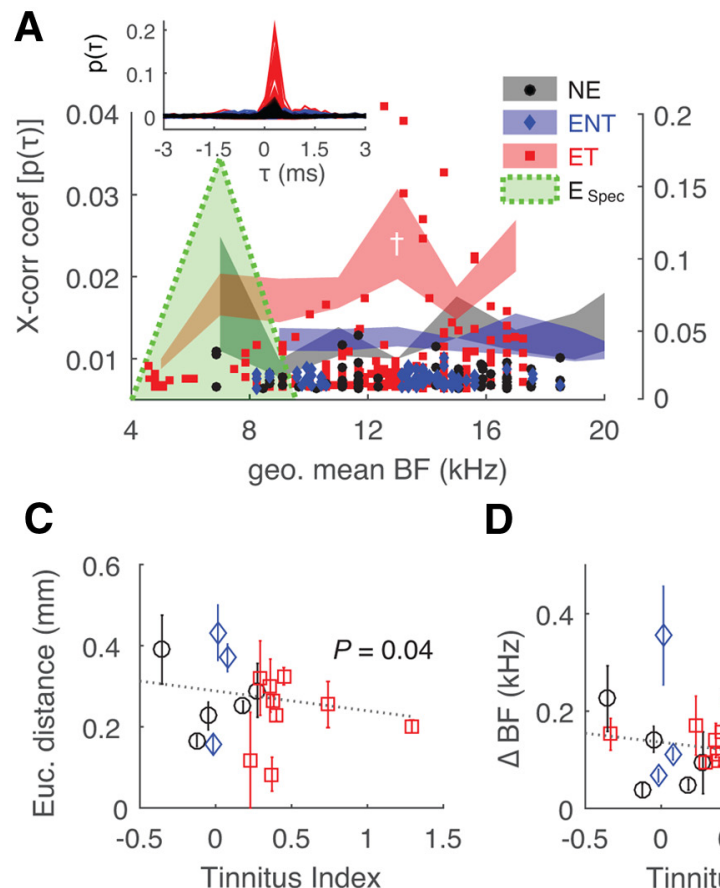

B

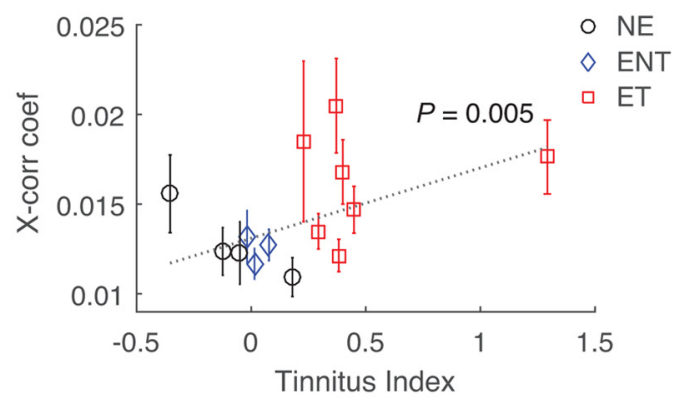

E

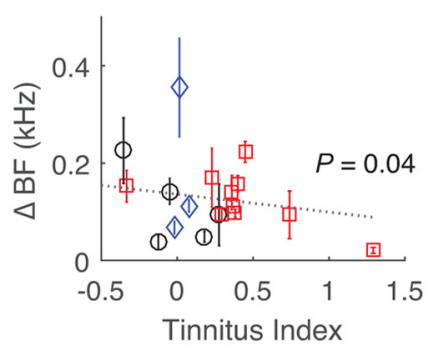

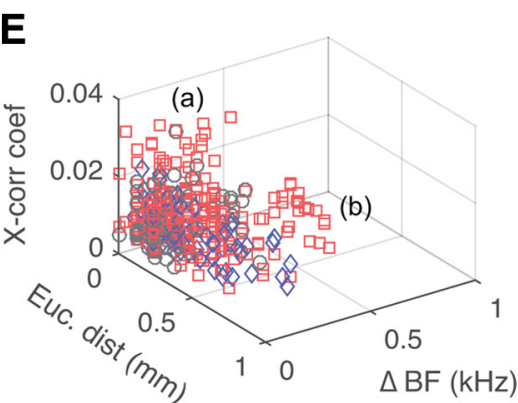

Figure 2. Synchrony of fusiform cells correlates with tinnitus behavior. $A$, Unit peak cross-correlation ( $x$-corr) coefficient as a function of geometric mean BFs of paired fusiform cells. Left, $y$-axis, Mean data; right, $y$-axis, individual data. $E_{\text {spe, }}$ Noise-exposure spectrum; post hoc, ${ }^{*} p<0.05$ from all groups, ${ }^{\dagger} p<0.05$ from ET/ENT. Inset, $x$-corr histograms $[p(\tau)]$ of all units in each group superimposed as functions of time lag $(\tau) . B, X$-corr of paired fusiform cells with geometric mean BFs in the 8,12 , and $16 \mathrm{kHz}$ bins ( $\pm 2 \mathrm{kHz}$ ) correlate with tinnitus indices at corresponding frequencies. $C, D$, Correlations of pairwise Euclidean distance between recording sites and pairwise differences in $B F(\triangle B F)$ with tinnitus index. $E$, Euclidean and $B F$ distance as functions of $x$-corr. (a) and (b) denote two distinct patterns of unit spatial distributions in tinnitus.

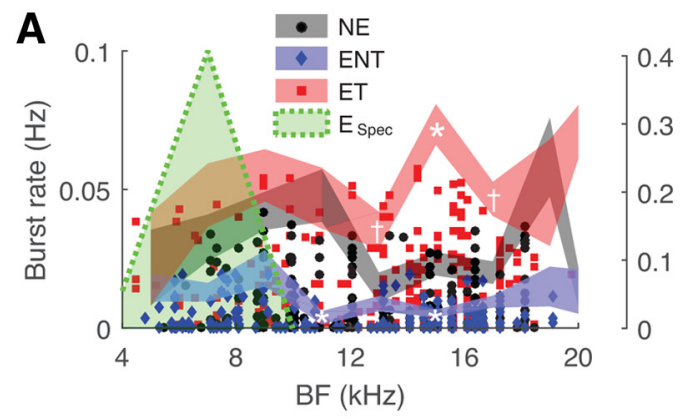

C

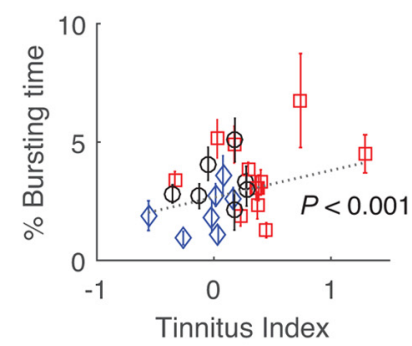

D

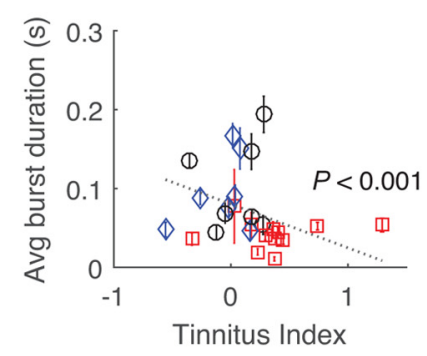

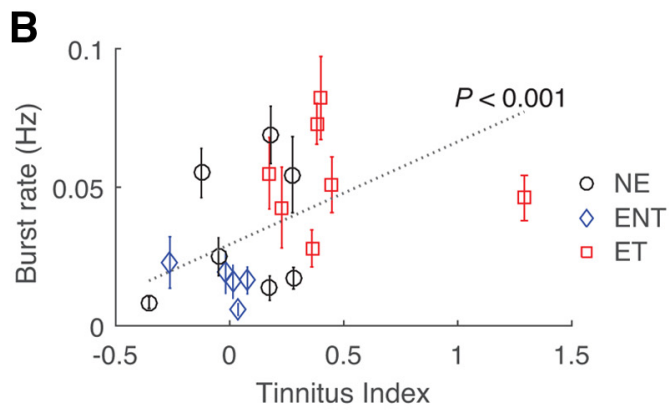

E

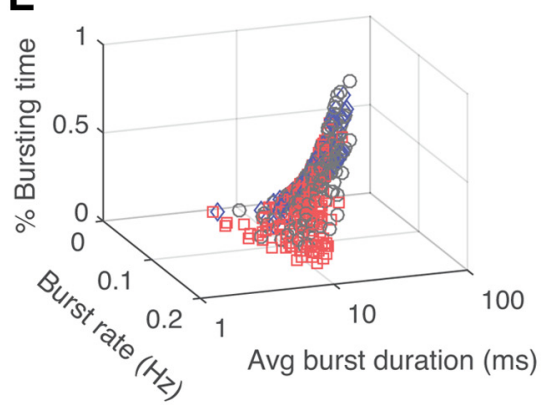

Figure 3. Bursting of fusiform cells correlates with tinnitus behavior. $A$, Spontaneous burst rate as a function of BF. Left, $y$-axis, Mean data; right, $y$-axis, individual data. $B$, Correlation of burst rate with tinnitus index. $\boldsymbol{C}, \boldsymbol{D}$, Correlations of percentage bursting time and average burst duration with tinnitus index. $\boldsymbol{E}$, Percentage bursting time and average burst duration as functions of burst rate.

correlates appear in tonotopic regions of the DCN that overlap the animal's tinnitus spectra, suggesting that the phantom percept is encoded in fusiform cell spontaneous activity. Although most studies focus on cortical synchrony, in which correlations between single-unit and EEG recordings are accessible (Singer, 1993), there is evidence from the somatosensory system that perceptual binding involves diffuse subcortical regions (Nicolelis et al., 1995). Thus, increased synchrony in fusiform cells likely also contributes to coding of the tinnitus percept. The present findings emphasize the role of DCN fusiform cells in tinnitus induction and suggest a bottom-up process of information transfer. SFR elevation in fusiform cells after noise trauma may influence their output targets in the IC (Manzoor et al., 2013) or thalamus (Malmierca et al., 2002; Kalappa et al., 2014). Likewise, neural synchrony may propagate across brain re- 
A

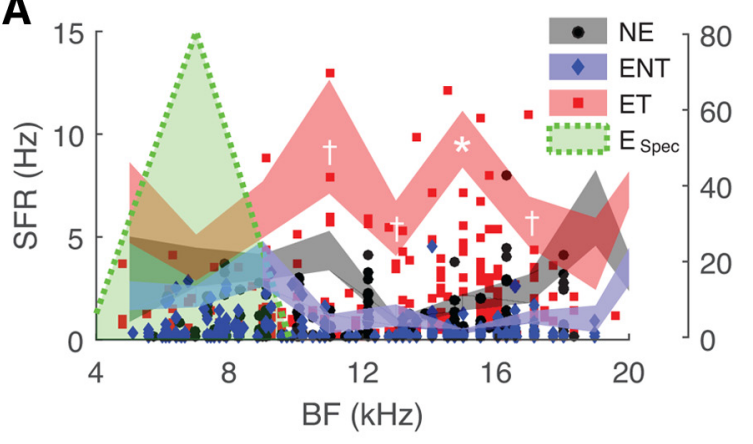

C

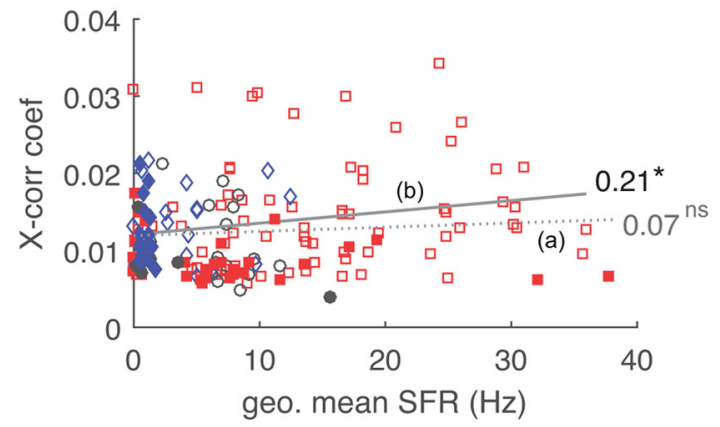

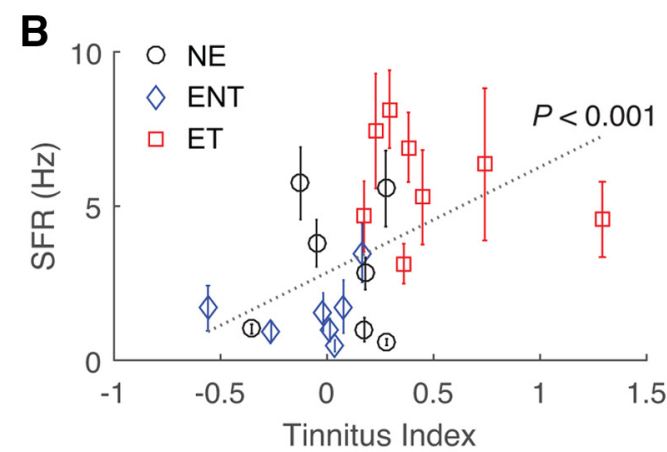

D

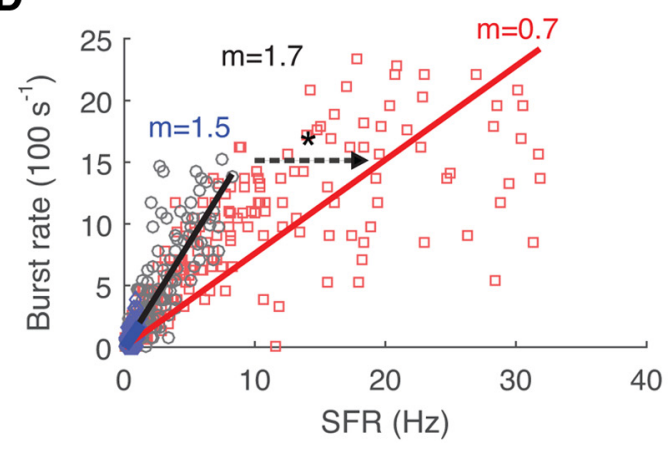

Figure 4. Synchrony, bursting, and SFR are independent correlates of tinnitus. $A$, Fusiform cell SFR as a function of BF. Left, $y$-axis, Mean data; right, $y$-axis, individual data. $B$, Correlation of SFR with tinnitus index. C, Correlations of peak cross-correlation coefficients with geometric mean SFRs of paired units. (a), Correlation of all unit pairs; (b), correlation of low-distance unit pairs. ${ }^{*} p<$ 0.05. $\boldsymbol{D}$, Correlations of burst rate with SFR. Correlation slopes $(m)$ are shown for each group. The arrow indicates slope difference. ${ }^{*} p<0.001$.

gions (Kumar et al., 2010) to institute increased synchrony and bursting in the IC (Bauer et al., 2008).

\section{Neural synchrony and tinnitus induction in fusiform cells}

Several mechanisms may regulate DCN synchrony. Fusiform cells receive granule cell-parallel fiber synapses on their apical dendrites. The noncochlear, excitatory projections to granule cells undergo homeostatic upregulation (Zeng et al., 2012) and contribute to longterm enhancement of fusiform cell excitability in tinnitus (Dehmel et al., 2012; Koehler and Shore, 2013). In the cerebellum, a structural analog of the DCN, principal neurons are synchronized by common granule cell inputs, an effect that decays as pairwise distance increases (highest synchrony $<300 \mu \mathrm{m}$; Heck et al., 2007). Consistent with the present study, in low-distance pairs, neural synchrony was dependent on firing rate (de la Rocha et al., 2007). Similarly, increased fusiform cell synchrony is likely driven by increased parallel fiber excitation [Fig. 4C, (b)]. However, increased parallel fiber activity can also potentiate a class of inhibitory interneurons, Golgi cells, to entrain additional parallel fibers into synchronized firing (Vos et al., 1999; Yaeger and Trussell, 2015). Consequently, this would widen the spatial distribution of a synchronized fusiform-cell network [Fig. 2E, (b)], because a single parallel fiber can traverse hundreds of microns (Mugnaini et al., 1980) and is unlikely to synapse onto nearby fusiform cells (Roberts and Trussell, 2010). In addition, parallel fiber-fusiform cell synapses exhibit spike timingdependent plasticity (STDP; Tzounopoulos et al., 2004), which can also regulate neural synchrony (Nowotny et al., 2003). The recent report of inverted STDP in fusiform cells after noise-induced tinnitus (Koehler and Shore, 2013) suggests that increased synchrony can emerge from altered circuit plasticity.

Along with increased excitation and altered plasticity, downregulation of glycinergic synapses on fusiform cells (Wang et al., 2009), as well as intrinsic changes in membrane kinetics (Pilati et al., 2012; Li et al.,
2015), drive fusiform cells to higher excitability. Increased bursting and altered burst duration have been associated with reduced high-voltageactivated potassium currents in fusiform cells (Pilati et al., 2012) and cerebellar principal neurons (McKay and Turner, 2004). Together, these reports suggest that tinnitus induction involves multiple processes: increased excitation (Dehmel et al., 2012), reduced inhibition (Wang et al., 2009), altered synaptic plasticity (Koehler and Shore, 2013), and intrinsic excitability (Li et al., 2015).

\section{Tinnitus resilience: the non-tinnitus animals}

Tinnitus does not always occur after auditory insult (König et al., 2006). In animal models, only 50-70\% of noise-exposed animals develop behavioral evidence of tinnitus (Koehler and Shore, 2013; Kalappa et al., 2014; Li et al., 2015). Tinnitus and non-tinnitus animals receive the same amount of measurable auditory nerve damage, reflected by equivalent threshold shifts (Fig. 1E,F) and suprathreshold wave I amplitudes (Fig. $1 H, I$ ). Although it is unknown whether cellular differences exist in the cochlea (Kujawa and Liberman, 2009), physiological signatures of tinnitus and nontinnitus are distinctive in fusiform cells: non-tinnitus animals exhibit significantly lower burst rates and SFRs compared with unexposed controls (Fig. 3A). This is consistent with the findings of Li et al. (2015) of more hyperpolarized fusiform cell resting membrane potentials in non-tinnitus compared with normal-hearing animals, thereby lowering spike incidence.

Few studies have focused on neural changes in non-tinnitus animals, but their opposing plasticity (lower burst rate and SFR) than both tinnitus and control animals suggests a mechanism of tinnitus resilience. Because non-tinnitus animals exhibit resilient phenotypes, there is an critical need to distinguish tinnitus from non-tinnitus animals. Many studies use noise exposure as a proxy for tinnitus, but, as demonstrated here, not all noise-exposed animals develop tinnitus, and those that do not develop tinnitus exhibit disparate physiological responses (Dehmel 
et al., 2012; Koehler and Shore, 2013; Li et al., 2015). Interpreting results from noise exposure studies that omit behavioral assessments may lead to erroneous conclusions.

Among various behavioral assessment techniques, the commonly used GPIAS provides a major advantage in its tinnitus spectrum estimation (Turner et al., 2006). However, GPIAS interpretation requires caution (Galazyuk and Hébert, 2015), because the fundamental assumption of how tinnitus affects gap and startle processing is currently under scrutiny (Fournier and Hébert, 2013). In the present study, accuracy of our behavioral outcomes was ensured by eliminating potential confounds: using a unilateral TTS model and performing prepulse inhibition to control for hearing impairment and ensuring stable startle reflexes to control for hyperacusis (Salloum et al., 2014). Moreover, physiological correlates including increased SFR (Brozoski et al., 2002; Kaltenbach et al., 2004; Dehmel et al., 2012), and as shown here, synchrony and bursting, can aid in ensuring consistency of GPIAS assessment across studies.

\section{References}

Bauer CA, Turner JG, Caspary DM, Myers KS, Brozoski TJ (2008) Tinnitus and inferior colliculus activity in chinchillas related to three distinct patterns of cochlear trauma. J Neurosci Res 86:2564-2578. CrossRef Medline

Berger JI, Coomber B, Shackleton TM, Palmer AR, Wallace MN (2013) A novel behavioural approach to detecting tinnitus in the guinea pig. J Neurosci Methods 213:188-195. CrossRef Medline

Brozoski TJ, Bauer CA, Caspary DM (2002) Elevated fusiform cell activity in the dorsal cochlear nucleus of chinchillas with psychophysical evidence of tinnitus. J Neurosci 22:2383-2390. Medline

de la Rocha J, Doiron B, Shea-Brown E, Josić K, Reyes A (2007) Correlation between neural spike trains increases with firing rate. Nature 448:802806. CrossRef Medline

Dehmel S, Pradhan S, Koehler S, Bledsoe S, Shore S (2012) Noise overexposure alters long-term somatosensory-auditory processing in the dorsal cochlear nucleus-possible basis for tinnitus-related hyperactivity? J Neurosci 32:1660-1671. CrossRef Medline

Eggermont JJ (2015) Animal models of spontaneous activity in the healthy and impaired auditory system. Front Neural Circuits 9:19. Medline

Elgoyhen AB, Langguth B, De Ridder D, Vanneste S (2015) Tinnitus: perspectives from human neuroimaging. Nat Rev Neurosci 16:632-642. CrossRef Medline

Engineer ND, Riley JR, Seale JD, Vrana WA, Shetake JA, Sudanagunta SP, Borland MS, Kilgard MP (2011) Reversing pathological neural activity using targeted plasticity. Nature 470:101-104. CrossRef Medline

Fournier P, Hébert S (2013) Gap detection deficits in humans with tinnitus as assessed with the acoustic startle paradigm: does tinnitus fill in the gap? Hear Res 295:16-23. CrossRef Medline

Galazyuk A, Hébert S (2015) Gap-prepulse inhibition of the acoustic startle reflex (GPIAS) for tinnitus assessment: current status and future directions. Front Neurol 6:88. Medline

Heck DH, Thach WT, Keating JG (2007) On-beam synchrony in the cerebellum as the mechanism for the timing and coordination of movement. Proc Natl Acad Sci U S A 104:7658-7663. CrossRef Medline

Kalappa BI, Brozoski TJ, Turner JG, Caspary DM (2014) Single unit hyperactivity and bursting in the auditory thalamus of awake rats directly correlates with behavioural evidence of tinnitus. J Physiol 592:5065-5078. CrossRef Medline

Kaltenbach JA (2011) Tinnitus: models and mechanisms. Hear Res 276:5260. CrossRef Medline

Kaltenbach JA, Zacharek MA, Zhang J, Frederick S (2004) Activity in the dorsal cochlear nucleus of hamsters previously tested for tinnitus following intense tone exposure. Neurosci Lett 355:121-125. CrossRef Medline

Koehler SD, Shore SE (2013) Stimulus timing-dependent plasticity in dorsal cochlear nucleus is altered in tinnitus. J Neurosci 33:19647-19656. CrossRef Medline

König O, Schaette R, Kempter R, Gross M (2006) Course of hearing loss and occurrence of tinnitus. Hear Res 221:59-64. CrossRef Medline

Kujawa SG, Liberman MC (2009) Adding insult to injury: cochlear nerve degeneration after "temporary" noise-induced hearing loss. J Neurosci 29:14077-14085. CrossRef Medline
Kumar A, Rotter S, Aertsen A (2010) Spiking activity propagation in neuronal networks: reconciling different perspectives on neural coding. Nat Rev Neurosci 11:615-627. CrossRef Medline

Li S, Kalappa BI, Tzounopoulos T (2015) Noise-induced plasticity of KCNQ2/3 and HCN channels underlies vulnerability and resilience to tinnitus. Elife 4:07242. CrossRef Medline

Malmierca MS, Merchán MA, Henkel CK, Oliver DL (2002) Direct projections from cochlear nuclear complex to auditory thalamus in the rat. J Neurosci 22:10891-10897. Medline

Manzoor NF, Gao Y, Licari F, Kaltenbach JA (2013) Comparison and contrast of noise-induced hyperactivity in the dorsal cochlear nucleus and inferior colliculus. Hear Res 295:114-123. CrossRef Medline

McKay BE, Turner RW (2004) Kv3 K+ channels enable burst output in rat cerebellar Purkinje cells. Eur J Neurosci 20:729-739. CrossRef Medline

Mugnaini E, Warr WB, Osen KK (1980) Distribution and light microscopic features of granule cells in the cochlear nuclei of cat, rat, and mouse. J Comp Neurol 191:581-606. CrossRef Medline

Nicolelis MA, Baccala LA, Lin RC, Chapin JK (1995) Sensorimotor encoding by synchronous neural ensemble activity at multiple levels of the somatosensory system. Science 268:1353-1358. CrossRef Medline

Noreña AJ, Eggermont JJ (2003) Changes in spontaneous neural activity immediately after an acoustic trauma: implications for neural correlates of tinnitus. Hear Res 183:137-153. CrossRef Medline

Nowotny T, Zhigulin VP, Selverston AI, Abarbanel HD, Rabinovich MI (2003) Enhancement of synchronization in a hybrid neural circuit by spike-timing dependent plasticity. J Neurosci 23:9776-9785. Medline

Pilati N, Large C, Forsythe ID, Hamann M (2012) Acoustic over-exposure triggers burst firing in dorsal cochlear nucleus fusiform cells. Hear Res 283:98-106. CrossRef Medline

Roberts MT, Trussell LO (2010) Molecular layer inhibitory interneurons provide feedforward and lateral inhibition in the dorsal cochlear nucleus. J Neurophysiol 104:2462-2473. CrossRef Medline

Salloum RH, Yurosko C, Santiago L, Sandridge SA, Kaltenbach JA (2014) Induction of enhanced acoustic startle response by noise exposure: dependence on exposure conditions and testing parameters and possible relevance to hyperacusis. PLoS One 9:e111747. CrossRef Medline

Singer W (1993) Synchronization of cortical activity and its putative role in information processing and learning. Annu Rev Physiol 55:349-374. CrossRef Medline

Turner JG, Brozoski TJ, Bauer CA, Parrish JL, Myers K, Hughes LF, Caspary DM (2006) Gap detection deficits in rats with tinnitus: a potential novel screening tool. Behav Neurosci 120:188-195. CrossRef Medline

Tzounopoulos T, Kim Y, Oertel D, Trussell LO (2004) Cell-specific, spike timing-dependent plasticities in the dorsal cochlear nucleus. Nat Neurosci 7:719-725. CrossRef Medline

Voigt HF, Young ED (1990) Cross-correlation analysis of inhibitory interactions in dorsal cochlear nucleus. J Neurophysiol 64:1590-1610. Medline

Vos BP, Maex R, Volny-Luraghi A, De Schutter E (1999) Parallel fibers synchronize spontaneous activity in cerebellar Golgi cells. J Neurosci 19: RC6(1-5). Medline

Wang H, Brozoski TJ, Turner JG, Ling L, Parrish JL, Hughes LF, Caspary DM (2009) Plasticity at glycinergic synapses in dorsal cochlear nucleus of rats with behavioral evidence of tinnitus. Neuroscience 164:747-759. CrossRef Medline

Weisz N, Müller S, Schlee W, Dohrmann K, Hartmann T, Elbert T (2007) The neural code of auditory phantom perception. J Neurosci 27:14791484. CrossRef Medline

Yaeger DB, Trussell LO (2015) Single granule cells excite Golgi cells and evoke feedback inhibition in the cochlear nucleus. J Neurosci 35:47414750. CrossRef Medline

Zacharek MA, Kaltenbach JA, Mathog TA, Zhang J (2002) Effects of cochlear ablation on noise induced hyperactivity in the hamster dorsal cochlear nucleus: implications for the origin of noise induced tinnitus. Hear Res 172:137-143. CrossRef Medline

Zeng C, Yang Z, Shreve L, Bledsoe S, Shore S (2012) Somatosensory projections to cochlear nucleus are upregulated after unilateral deafness. J Neurosci 32:15791-15801. CrossRef Medline

Zhang JS, Kaltenbach JA, Godfrey DA, Wang J (2006) Origin of hyperactivity in the hamster dorsal cochlear nucleus following intense sound exposure. J Neurosci Res 84:819-831. CrossRef Medline 